\title{
Physically injured patients described several forms of post-traumatic concerns
}

Zatzick DF, Kang SM, Ladson Hinton W, et al. Posttraumatic concerns: a patient-centered approach to outcome assessment after traumatic physical injury. Med Care 2001 Apr; 39:327-39.

\section{QUESTION: What are the post-traumatic concerns of physically injured patients?}

Design

Qualitative study.

\section{Setting}

University of California at Davis Medical Centre, USA.

\section{Patients}

97 English speaking survivors (age range 18-65 y, 56\% men) of motor vehicle crashes or assaults who were randomly selected from those admitted to the hospital. Patients who had sustained substantial head, spinal cord, and other severe injuries, or who had ongoing surgery were excluded.

\section{Methods}

Data were collected by face to face interviews during initial hospital stay, followed by telephone interviews at 1,4 , and 12 months after the traumatic injury. Interviews were used to collect demographic and individual characteristics, medical history, psychiatric symptoms, functional status, and health service use, and open ended questions were used to determine post traumatic illness experience. Qualitative data were analysed using content analysis by grouping content into categories that represented 7 domains. Inter-rater reliability of domain categorisation ranged from $0.70-0.83$.

\section{Main findings}

$99 \%$ of patients expressed $\geqslant 1$ concern. The most common concern was physical health which focused on concerns regarding bodily injury, pain, and self care. Second to physical health was the psychological domain which included expressions of post-traumatic distress such as anxiety and depression. The third domain was work and finance. This domain reflected concerns about employment, finances, housing, education, and food acquisition. The fourth domain was social, reflecting both positive and negative concerns regarding people in the patient's social network. Additional domains were legal and medical. Legal concerns focused on interactions with the police, courts, or other criminal justice agencies. Medical concerns reflected worries about interactions with providers and financing of care. Although mean number of concerns expressed per patient gradually decreased over time, psychological and work concerns increased from 1 month to 4 months. Increases and decreases in concerns were mirrored by similar increases and decreases in quantitative measures of post-traumatic stress and functional limitations.

\section{Conclusions}

Physically injured trauma survivors could clearly express concerns about their illness experience as they evolved over time. These concerns corresponded to other quantitative measures of illness severity.

\section{COMMENTARY}

The aim of this patient centred, qualitative inquiry by Zatzick et al was to develop a method of assessing, categorising, and following up the post-traumatic concerns of men and women who have survived traumatic physical injury. The reader is provided with insight into the needs, concerns, and meanings people attach to this profound life crisis. The findings of this research will enhance clinical practice by enabling a contextual understanding of a trauma patient's experiences that extends beyond biomedical concerns.

This research offers health professionals new knowledge that can be applied when caring for trauma patients. Few inquiries have studied the perspective of the trauma patient. The process and method of the study were clearly articulated and provided the reader with an understanding of how the researchers arrived at their findings.

Data were generated by face to face interviews during initial hospital stay, followed by telephone interviews at 1,4 , and 12 months. Patients' post-traumatic concerns were grouped into 7 categories: physical health, psychological concerns, employment and financial, social, legal, medical, and uncodable concerns. Trauma patients concerns' evolved over time, which indicates that this group of patients requires regular clinical reassessment. Clearly, the meaning of surviving a trauma for patients extends beyond the initial physical injuries to have a profound impact on other aspects of their lives.

The longitudinal nature of this study has merit because we are exposed to the transitional nature of trauma patients' concerns, and the complex person-environmental interactions that these people experience. This study could develop links to the transition theory emerging in the health literature that describes the sense of movement that people, particularly those recovering from physical assault, experience over time.

It seems important for health professionals to provide opportunities for trauma patients to openly communicate about their concerns on an ongoing basis. The acknowledgment of the trauma patient's evolving concerns by a health professional would be affirming and validating for them when they are struggling with the sudden and unexpected effects of a serious physical assault.

Through this study, the reader is sensitised to the transitional, evolving, and contextual complexity of the concerns of patients who survive serious trauma injuries, and as such the findings are of importance not only to medical and nursing clinicians, but also to the wider healthcare community.

Debbie Kralik RN, MN, PhD, MRCNA Royal District Nursing Service Glenside, South Australia, Australia 